\title{
Hiperglicemia en niños con leucemia linfoblástica aguda en tratamiento con L-asparaginasa
}

\author{
PAULA PALMA R. ${ }^{1}$, ISABEL FOLATRE B. ${ }^{2}$, MÓNICA KYONEN L. ${ }^{1}$, GUSTAVO CEA S. ${ }^{2}$, MANIA YILORM B. ${ }^{2}$, \\ PILAR MARTÍNEZ D. ${ }^{2}$
}

1. Químico Farmacéutico, Subdepartamento de Farmacia, Hospital Base Valdivia.

2. Médico Onco-Hematólogo Infantil, Instituto de Pediatría. Universidad Austral de Chile, y Unidad de Pediatría. Hospital Base Valdivia.

\begin{abstract}
Evaluation and characterization of hyperglycemia in children with acute lymphoblastic leukemia treated with L-asparaginase

Introduction: L-asparaginase (L-asp) is an antineoplastic agent that has among its adverse reactions, hyperglycemia. Each insulin molecule has three asparagine residues which are hydrolyzed by L-asp, decreasing insulin synthesis and resulting in hyperglycemia. The objective of this study is to describe the characteristics of hyperglycemia associated with the use of L-asp in children with Acute Lymphoblastic Leukemia (ALL). Patients and Method: A retrospective study that included review of medical records of all children admitted to Valdivia Center of the National Children's Antineoplastic Drug Program, between 2002 and 2009 was performed. Patient backgrounds were evaluated and those who were suspected to present adverse drug reactions were evaluated according to the causality classification of Karch and Lasagna. Results: 85 of 102 patients who met the inclusion criteria entered the study. The incidence of hyperglycemia was $6.74 \%$. The patients with hyperglycemia were those older than 9.5 years of age, with risk of obesity, diabetes, family history of diabetes mellitus and concomitant corticosteroid treatment. Conclusion: The characteristics of hyperglycemia in patients treated with L-asp coincide with those of the literature. Simultaneous administration of corticosteroids and L-asp make difficult to determine causality of L-asp, so further research is needed.
\end{abstract}

(Key words: Adverse reaction, L-asparaginase, acute lymphoblastic leukemia, hyperglycemia).

Rev Chil Pediatr 2013; 84 (4): 387-395

\section{RESUMEN}

Introducción: L-asparaginasa (L-asp), es un agente antineoplásico, entre cuyos efectos adversos se describe hiperglicemia dado que cada molécula de insulina tiene tres residuos de asparragina que son hidrolizados por

Recibido el 18 de septiembre de 2012, devuelto para corregir el 20 de noviembre de 2012, segunda versión 18 de febrero de 2013, aceptado para publicación el 9 de julio de 2013.

Este trabajo cumple con los requisitos sobre consentimiento /asentimiento informado, comité de ética, financiamiento, estudios animales y sobre la ausencia de conflictos de intereses según corresponda. 
L-asp, produciendo disminución de la síntesis de insulina con la consecuente hiperglicemia. Nuestro objetivo fue describir las características de la hiperglicemia asociada al uso de L-asp en niños en tratamiento por LLA. Pacientes y Método: Estudio retrospectivo que incluyó la revisión de historias clínicas de todos los niños, ingresados al Centro Valdivia, del Programa Infantil Nacional de Drogas Antineoplásicas, entre los años 2002 y 2009. Se evaluaron los antecedentes de los pacientes y aquellos que resultaron sospechosos de reacción adversa a medicamentos, se evaluaron según el algoritmo de causalidad de Karch y Lasagna. Resultados: Ingresaron al estudio 85 de 102 pacientes que reunieron los criterios de inclusión. La incidencia de hiperglicemia fue $6,74 \%$. Los pacientes que presentaron hiperglicemia fueron aquellos con edad mayor a 9,5 años, riesgo de obesidad, antecedentes familiares de diabetes Diabetes Mellitus y tratamiento concomitante con corticoides. Conclusión: Las características de los pacientes con hiperglicemia en tratamiento con L-asp coinciden con los de la literatura. La administración simultánea de corticoides y L-asp dificulta la determinación de causalidad de L-asp, por lo que es importante que se continúe en el futuro con esta línea de investigación.

(Palabras clave: Reacción adversa, L-asparaginasa, leucemia linfoblástica aguda, hiperglicemia).

Rev Chil Pediatr 2013; 84 (4): 387-395

\section{Introducción}

El Hospital Base Valdivia es un centro de referencia nacional para el estudio y tratamiento de pacientes portadores de cáncer, beneficiarios del Programa Infantil Nacional de Drogas Antineoplásicas (PINDA) procedentes de las regiones X, XI, XII y XIV. Anualmente se reciben entre 15 y 24 pacientes con diagnóstico de leucemia linfoblástica aguda (LLA). PINDA en sus protocolos de inducción y consolidación de la remisión para LLA, considera quimioterapia combinada con diversos agentes antineoplásicos, entre ellos L-asparaginasa (Lasp), enzima de origen bacteriano, que puede ser obtenida naturalmente a partir de Escherichia coli y Erwinia chrysantemi ${ }^{1}$. Además de estas formas nativas, existe PEG-L-asparraginasa (PEG-asp), que es L-asp proveniente de E. coli conjugada con un grupo polietilenglicol (PEG) y desarrollada con el objeto de mejorar características farmacocinéticas.

Casi todos los tejidos normales sintetizan asparagina en cantidades suficientes para la síntesis proteica. Algunas neoplasias, como LLA necesitan una fuente exógena de dicho aminoácido, ya que poseen muy bajos niveles de asparagina sintetasa, enzima que cataliza la producción de este aminoácido ${ }^{2}$. Los linfoblastos obtienen por lo tanto asparagina a partir del plasma del huésped. L-asp hidroliza la asparagina a ácido aspártico y amonio resultando en depleción de la asparragina sérica y muerte de los linfoblastos ${ }^{2,3}$.
Las Reacciones adversas a medicamentos (RAM) provocadas por L-asp tienen una incidencia variable, 5-33\% de acuerdo a datos internacionales ${ }^{4,5}$. Entre las más frecuentemente observadas se encuentran las reacciones de hipersensibilidad con una incidencia de $0-45 \%{ }^{6}$, desequilibrio de las proteínas de la coagulación $1-37 \%$, alteraciones neurológicas tales como somnolencia, agitación, depresión, alucinaciones, desorientación, convulsiones y coma, $0-25 \%{ }^{9,10}$, anormalidades de la función hepática $0-3 \%$, caracterizada por aumento de transaminasas, fosfatasas alcalinas, bilirrubina y cambios en el metabolismo de los lípidos ${ }^{11,12}$, pancreatitis $0-16 \%{ }^{13}$ y alteraciones en el metabolismo de la glucosa ocasionando hiperglicemia y diabetes mellitus $2,5-23 \%{ }^{14}$.

Roberson y cols ${ }^{15}$, describen que $10-15 \%$ de los pacientes con LLA presentaron hiperglicemia, en asociación al uso de L-asp, corticoides e infecciones. Por otra parte, se ha demostrado que la hemoglobina glicosilada basal en pacientes con diagnóstico de LLA es mayor que la observada en pacientes no leucémicos, especialmente cuando el recuento leucocitario es mayor a $20.000 / \mathrm{mm}^{3}$ probablemente asociado a una alteración en la producción de citoquinas por disregulación inmune.

L-asp produce hiperglicemia mediante hidrólisis de los residuos de asparagina de la insulina; cada molécula tiene tres residuos de ésta, produciendo disminución de su síntesis tanto como de su secreción en las células beta 
del páncreas. También altera la función de los receptores de ésta.

Los corticoides estimulan la gluconeogénesis y causan disminución de la utilización periférica de la glucosa. El uso combinado de L-asp y corticoides sinérgicamente produce hiperglicemia y pancreatitis ${ }^{15}$, por lo que se recomienda medir glicemia y amilasemia pre y post administración de L-asp. Baillargeon y $\operatorname{cols}^{16}$, describieron hiperglicemia en $4-15 \%$ de los niños en tratamiento combinado con L-asp y corticoides por LLA.

La detección y tratamiento tempranos de la hiperglicemia se consideran un paso clave en la prevención de complicaciones agudas tales como síndrome hiperosmolar hiperglicémico no cetoacidótico y cetoacidosis diabética ${ }^{17}$.

La determinación de la causalidad de una sospecha de RAM, se basa en los criterios de la Tabla de Decisiones de Karch y Lasagna (preguntas del Algoritmo del Sistema Español de Fármacovigilancia) y en las Categorías de Causalidad de la OMS que asignan puntaje a las sospechas de RAM y clasifica como definitivas ( $\geq 8$ puntos) a aquellas alteraciones que guardan relación temporal con la administración del medicamento y no pueden ser atribuídas a la enfermedad u otro fármaco; RAM probables (6-7 puntos) alteraciones, que si bien guardan relación temporal con la administración del fármaco, no podrían ser explicadas por la enfermedad u otro medicamento administrado, ya que responden favorablemente a la suspensión de la droga. En la categoría de posibles (4-5 puntos), caen las alteraciones observadas después de la exposición al medicamento, que podrían ser causadas por la enfermedad de base o la administración de otra droga y en las cuales no hay información respecto a la respuesta a la suspensión del fármaco. Las RAM condicionales/no clasificadas (1-3 puntos), son aquellas en las cuales es necesario contar con mayores antecedentes para una apropiada evaluación y las improbables $(<$ 1 punto), no guardan relación temporal con la exposición al fármaco y pueden ser atribuidas a la enfermedad de base o a otro fármaco.

El objetivo de este trabajo es describir las características de la hiperglicemia asociada al uso de L-asp en niños en tratamiento por LLA.

\section{Pacientes y Método}

Estudio retrospectivo que incluyó la revisión de historias clínicas de todos los niños con diagnóstico y tratamiento por LLA, ingresados al centro PINDA Valdivia, entre el 1 de enero de 2002 y el 31 de diciembre de 2009, ambas fechas incluidas.

Durante el tratamiento según Protocolo de Inducción PINDA para LLA, se administró L-asp en dosis de $5.000 \mathrm{UI} / \mathrm{m}^{2}$ de superficie corporal, vía endovenosa, día por medio por 8 veces. El Protocolo de Reinducción consideró el uso de L-asp en dosis de $10.000 \mathrm{UI} / \mathrm{m}^{2}$ de superficie corporal, día por medio, vía endovenosa, por 4 veces y $10.000 \mathrm{UI} / \mathrm{m}^{2}$ de superficie corporal en dosis única endovenosa, en los bloques de recidiva.

Se definió como ayuno a la falta de ingesta calórica durante al menos $8 \mathrm{~h}$; normoglicemia a glicemia en ayunas $100-125 \mathrm{mg} / \mathrm{dl}$; Intolerancia a la glucosa a glicemia entre 140 y 199 $\mathrm{mg} / \mathrm{dl}$ a las $2 \mathrm{~h}$ después de una carga estandarizada de glucosa y Diabetes Mellitus, como un grupo de enfermedades metabólicas caracterizado por hiperglicemia crónica que resulta de defectos en la secreción de insulina, acción de la insulina o ambos ${ }^{18}$.

Durante el protocolo de Inducción se administró prednisona $60 \mathrm{mg} / \mathrm{m}^{2} /$ día por 28 días; en Reinducción, dexametasona $10 \mathrm{mg} / \mathrm{m}^{2} /$ día por 21 días; y en Recaída, dexametasona $20 \mathrm{mg}$ / $\mathrm{m}^{2} /$ día por cinco días en cada bloque.

Se evaluaron las fichas clínicas de todos los pacientes portadores de LLA menores de 14 años 11 meses y 28 días de edad al momento del diagnóstico. Se excluyeron del estudio pacientes fallecidos antes de iniciar tratamiento con L-asp, portadores conocidos de Diabetes Mellitus, pacientes trasladados desde o hacia otro centro asistencial durante el tratamiento con L-asp, pacientes tratados con PEG-asp y los pacientes cuyas fichas clínicas no estuvieron disponibles al momento del estudio. Se obtuvo datos de alteraciones clínicas, tales como polidipsia, poliuria, polifagia y de laboratorio, tales como glicemia, pruebas de función pancreática, hepática y electrolitos sanguíneos, durante el tiempo que el paciente estuvo en tratamiento con L-asp. 
Se seleccionó aquellas alteraciones sospechas de RAM y fueron evaluadas según el algoritmo de causalidad del Sistema Español de Farmacovigilancia ${ }^{19}$; las sospechas de RAM que resultaron ser probables, fueron ingresadas al estudio de episodios hiperglicémicos en relación al uso de L-asp.

\section{Resultados}

Entre el 9 de enero del año 2002 y el 1 de diciembre del año 2009, ingresaron al centro PINDA un total de 102 niños con diagnóstico de LLA.

Se enrolaron en el estudio 85 de 102 pacientes que reunieron los criterios de inclusión; 17 pacientes fueron excluidos: 3 por traslado a otro centro asistencial y 14 por falta de disponibilidad de las historias clínicas al momento de la revisión.

De los 85 pacientes, 73 presentaron 89 episodios de hiperglicemia, registrándose 1,2 episodios de hiperglicemia por paciente. Cada episodio fue evaluado mediante el Algoritmo de Causalidad del Sistema Español de Farmacovigilancia, resultando en la categoría Probable, 6, en la Posible se ubicaron 21, en la Condicional se registraron 20, y en la Improbable 42 episodios.

La incidencia de episodios de hiperglicemia secundarios al tratamiento con L-asp en niños con LLA, fue de 6,74\% (6 de 89 episodios de hiperglicemia).

Los antecedentes generales, protocolos de tratamiento y valores de laboratorio de los pacientes con RAM observados según categorización de RAM se muestran en la tabla 1. En la tabla 2 se describen las características de cada uno de los 6 pacientes con RAM probable y en la tabla 3 , se presentan las características clínicas y de laboratorio de 6 pacientes con RAM probable.

Dos de 6 pacientes recibieron tratamiento para la RAM. El régimen diabético fue la primera medida instaurada para controlar la hiperglicemia. En el episodio inicial, además se administró insulina cristalina s.c. por $48 \mathrm{~h}$, para controlar glicemias $>250 \mathrm{mg} / \mathrm{dL}$ y Glibencla-

Tabla 1. Distribución de las categorías de RAM según variables

\begin{tabular}{|c|c|c|c|c|c|}
\hline \multicolumn{2}{|l|}{ Variables } & $\begin{array}{c}\text { Probable } \\
n=6\end{array}$ & $\begin{array}{l}\text { Posible } \\
n=21\end{array}$ & $\begin{array}{c}\text { Condicional } \\
n=20\end{array}$ & $\begin{array}{c}\text { Improbable } \\
n=42\end{array}$ \\
\hline \multicolumn{2}{|l|}{ Edad (años) } & $9,6 \pm 4,8$ & $6,2 \pm 4,1$ & $7,1 \pm 4,1$ & $5,59 \pm 3,3$ \\
\hline \multirow[t]{2}{*}{ Género } & Masculino & 3 & 11 & 9 & 23 \\
\hline & Femenino & 3 & 10 & 11 & 19 \\
\hline \multirow[t]{4}{*}{ IMC } & $p<10$ & 0 & 0 & 0 & 5 \\
\hline & p 10 y $p<85$ & 2 & 11 & 11 & 22 \\
\hline & $p 85$ у $p<95$ & 2 & 6 & 8 & 7 \\
\hline & $p \geq 95$ & 2 & 4 & 1 & 8 \\
\hline \multicolumn{2}{|l|}{ Antecedentes familiares DM } & 3 & 7 & 7 & 14 \\
\hline \multirow[t]{4}{*}{ Etapa tratamiento } & Inducción & 3 & 14 & 15 & 38 \\
\hline & Consolidación & 0 & 2 & 1 & 0 \\
\hline & Reinducción & 1 & 2 & 3 & 2 \\
\hline & Recaída & 2 & 3 & 1 & 2 \\
\hline \multirow[t]{4}{*}{ Fármacos concomitantes } & Prednisona & 2 & 8 & 7 & 17 \\
\hline & Dexametasona & 1 & 4 & 4 & 3 \\
\hline & Predn./Furosemida & 1 & 6 & 8 & 21 \\
\hline & Dexa./Furosemida & 2 & 3 & 1 & 1 \\
\hline \multicolumn{2}{|l|}{ Síndrome Down } & 0 & 2 & 2 & 0 \\
\hline \multicolumn{2}{|l|}{ Glicemia (mg/dL) } & $160,4 \pm 225,2$ & $109,1 \pm 31,6$ & $119,9 \pm 55,6$ & $103,6 \pm 36,9$ \\
\hline \multicolumn{2}{|l|}{ Amilasemia (UI/L) } & $69,9 \pm 34,3$ & $65,5 \pm 34,0$ & $51,7 \pm 28,3$ & $49,0 \pm 21,5$ \\
\hline \multicolumn{2}{|l|}{ Potasemia (mEq/L) } & $4,0 \pm 0,5$ & $3,9 \pm 0,5$ & $4,0 \pm 0,7$ & $4,1 \pm 0,6$ \\
\hline
\end{tabular}

RAM: Reacción adversa a medicamentos. DM: Diabetes mellitus. IMC: Índice de masa corporal. 
mida por 14 días. En el segundo episodio, con glicemia en ayunas de $1.560 \mathrm{mg} / \mathrm{dl}$, además del regimen diabético e insulina cristalina, se administró insulina NPH, 6 días después del inicio de la RAM, hasta que las glicemias se normalizaron.

Tabla 2. Distribución de los 6 pacientes con RAM probable según sus características

\begin{tabular}{|c|c|c|c|c|c|c|c|c|c|c|}
\hline Caso & $\begin{array}{l}\text { Edad } \\
\text { (años) }\end{array}$ & Sexo & IMC & $\begin{array}{c}\text { Antece- } \\
\text { dentes } \\
\text { familiares } \\
\text { DM }\end{array}$ & $\begin{array}{c}\text { Etapa } \\
\text { tratamiento }\end{array}$ & $\begin{array}{c}\text { Fármacos } \\
\text { concomitantes }\end{array}$ & $\begin{array}{c}\text { Glicemia } \\
\text { basal } \\
\text { (mg/dL) }\end{array}$ & $\begin{array}{c}\text { Glicemia } \\
\text { con RAM } \\
\text { (mg/dL) }\end{array}$ & $\begin{array}{l}\text { Amila- } \\
\text { semia } \\
\text { (UI/L) }\end{array}$ & Síntomas \\
\hline 1 & 14 & $\mathrm{~F}$ & $=\mathrm{p} 95$ & Sí & Reinducción & Dexametasona & 85 & $\begin{array}{c}451 \\
* \text { Glucosuria } \\
++100 \\
\text { mg/dL }\end{array}$ & - & $\begin{array}{l}\text { Ardor epigástrico } \\
\text { Polidipsia } \\
\text { Poliuria } \\
\text { Parestesias en } \\
\text { extremidad } \\
\text { inferior izquierda }\end{array}$ \\
\hline 2 & 13,5 & M & $\begin{array}{l}\text { p } 85 \text { y } \\
<\text { p } 95\end{array}$ & Sí & Inducción & $\begin{array}{l}\text { Prednisona/ } \\
\text { furosemida }\end{array}$ & 86 & 1.560 & 132,6 & $\begin{array}{l}\text { Acidosis metabólica } \\
\text { parcialmente compensada, } \\
\text { no en rangos de corregir } \\
\text { Decaimiento } \\
\text { Obnubilación } \\
\text { Polidipsia } \\
\text { Mucosas secas }\end{array}$ \\
\hline 3 & 2,9 & M & $=\mathrm{p} 95$ & No & Recaída & $\begin{array}{l}\text { Dexametasona/ } \\
\text { furosemida }\end{array}$ & 97 & 155 & 99 & Sin síntomas \\
\hline 4 & 6,5 & $\mathrm{~F}$ & $\begin{array}{l}\text { p } 10 y \\
<p 85\end{array}$ & Sí & Inducción & Prednisona & 88 & 140 & 51,5 & Sin síntomas \\
\hline 5 & 6,75 & $\mathrm{~F}$ & $\begin{array}{l}\text { p } 85 \text { y } \\
<\text { p } 95\end{array}$ & No & Inducción & Prednisona & 89 & 219 & - & Sin síntomas \\
\hline 6 & 14 & $M$ & $\begin{array}{l}\text { p } 10 y \\
<p 85\end{array}$ & No & Recaída & $\begin{array}{l}\text { Dexametasona/ } \\
\text { furosemida }\end{array}$ & 87 & 173 & 91,3 & Sin síntomas \\
\hline
\end{tabular}

RAM: Reacción adversa a medicamentos. IMC: Índice de masa corporal. DM: Diabetes mellitus.

Tabla 3. Características Clínicas y de Laboratorio de 6 pacientes con RAM probable

\begin{tabular}{|c|c|c|c|c|c|c|}
\hline$\stackrel{n}{\text { pac }}$ & $\begin{array}{l}\text { Tipo alteración } \\
\text { glicemia }\end{array}$ & Duración & Requerimiento tratamiento & $\begin{array}{l}\text { Tiempo de } \\
\text { tratamiento }\end{array}$ & $\begin{array}{l}\text { Evaluación } \\
\text { endocrino }\end{array}$ & $\begin{array}{c}\text { Cumple } \\
\text { criterios DM* }\end{array}$ \\
\hline 1 & En ayuno & 4 días & Ninguno & $\mathrm{n} / \mathrm{a}$ & $\mathrm{n} / \mathrm{a}$ & No \\
\hline 2 & En ayuno & 7 días & $\begin{array}{l}\text { Insulina cristalina } 8 \cup \text { sc; si glicemia } \\
\text { es }>250 \mathrm{mg} / \mathrm{dl} \text {. Régimen diabético, } \\
\text { Glibenclamida } 2,5 \mathrm{mg}\end{array}$ & 1 mes & sí & No \\
\hline 3 & $\begin{array}{l}\text { Glicemias en } \\
\text { ayuno, con } \\
\text { síntomas de DM }\end{array}$ & 1 mes & $\begin{array}{l}\text { Insulina Cristalina } 6 \text { UI bolo }(0,1 \mathrm{UI} / \mathrm{kg}) \\
\text { luego } 0,1 \mathrm{UI} / \mathrm{kg} / \mathrm{h} \text { en } \mathrm{BIC} \\
\text { Alimentación } 2.000 \text { cal: } 50 \% \text { cal como } \\
\mathrm{HC} \text {--- } 60 \mathrm{~g} \text { x } 4 \text { veces en } 4 \text { comidas } \\
\text { isoglucídicas; hipograso; agua libre } \\
\text { Insulina cristalina } 15 \mathrm{UI} \text { sc } 30 \text { min antes } \\
\text { de cada comida } \\
\text { Insulina lenta NPH } 30 \mathrm{UI}\end{array}$ & $>1$ mes & Sí & Sí \\
\hline 4 & $\begin{array}{l}\text { Intolerancia a la } \\
\text { glucosa }\end{array}$ & 1 mes & $\mathrm{n} / \mathrm{a}$ & $\mathrm{n} / \mathrm{a}$ & No & No \\
\hline 5 & En ayuno & 7 días & $\mathrm{n} / \mathrm{a}$ & $\mathrm{n} / \mathrm{a}$ & No & No \\
\hline 6 & En ayuno & 10 días & $\mathrm{n} / \mathrm{a}$ & n/a & No & No \\
\hline
\end{tabular}

DM: Diabetes mellitus. 


\section{Discusión}

Nuestro estudio detectó 89 episodios de hiperglicemia en 73 pacientes pediátricos tratados por LLA (RAM Probables, 6; Posibles 21; Condicionales 20 e Improbables 42), con una frecuencia de hiperglicemia fue de $82 \%$ para este grupo de pacientes. Este valor es más alto que lo informado en la literatura para niños con LLA tratados con L-asp y corticoides (4$15 \%)^{20}$, probablemente debido a la definición de hiperglicemia, que en esta investigación fue $\geq 125 \mathrm{mg} / \mathrm{dl}$ y considerando que en la mayoría de los estudios se definió hiperglicemia como glicemias en ayunas $\geq 200 \mathrm{mg} / \mathrm{dl}^{15,16,20,21}$.

La incidencia de hiperglicemia secundaria a L-asp (RAM Probables) fue 6,74\% (6 de 89 episodios) valor que coincide con lo reportado en otros trabajos en niños tratados con L-asp por LLA $(2,5-23 \%)^{14}$

Análisis de causalidad según el Algoritmo del Sistema Español de Farmacovigilancia. Secuencia temporal: en 4 de 6 casos probables el intervalo de administración de L-asp fue compatible con la aparición de hiperglicemia, en un período que fluctuó desde $24 \mathrm{~h}$ a algunas semanas. En la literatura se describen episodios de hiperglicemia dentro de los primeros 30 días de tratamiento con L-asp ${ }^{22}$. Por otra parte, en 2 de 6 casos clasificados como probables, se encontró valores de elevados de glicemia previos a la administración de L-asp $(118,2 \pm 28,2 \mathrm{mg} / \mathrm{dl})$ y que se mantuvieron elevados en relación a la administración de L-asp, por lo cual en estos casos se consideró que el intervalo de administración no fue totalmente compatible y puede ser explicado por el uso de corticoides.

Conocimiento previo de la RAM: éste se encuentra descrito en publicaciones nacionales e internacionales, en los que se han analizado los mecanismos probables por los cuales el fármaco produce hiperglicemia en los pacientes expuestos.

Efecto de la retirada: cabe destacar que en ningún paciente se suspendió el tratamiento con L-asp, pese a la reacción adversa, ya que el costo de suspender el fármaco es mayor que el beneficio de mantener la administración de L-asp, tratando la hiperglicemia.
Reexposición: hubo reexposición al fármaco en todos los pacientes, por las razones anteriormente mencionadas. En 4 de 6 RAM probables hubo hiperglicemia luego de la re administración de la droga; en 1 de 6 RAM probables ésta fue similar con especialidades diferentes, conteniendo el mismo principio activo del fármaco (L-asp); en 1 de 6 episodios no hubo re exposición. Pui y cols. ${ }^{20}$, describen que la mayoría de los pacientes que hicieron hiperglicemia no empeoraron ni presentaron elevación en los valores de glicemia luego de la reexposición a L-asp.

Existencia de una causa alternativa: en la mayoría de los pacientes hubo una explicación alternativa a la relación causal entre la RAM y L-asp, que presentó igual o menor similitud; en este estudio fue principalmente la administración concomitante de corticoides que han sido asociados a la aparición de hiperglicemia. Roberson y cols. ${ }^{15}$ Baillargeon y cols. ${ }^{16}$, tanto como Lowas y cols. ${ }^{19}$, reportan que el uso de corticoides en concomitancia con L-asp representa una limitante en la determinación de la causalidad de hiperglicemia, ya que en los protocolos para el tratamiento de LLA se administra estos fármacos de forma simultánea y con gran impacto en la inducción de la remisión medular. La frecuencia de hiperglicemia secundaria a corticoides es muy variable, 1 a 46\% según estudios observacionales ${ }^{23,24}$ y característicamente provoca un aumento, en particular, de la glicemia postprandial, mínima elevación de la glicemia basal e insensibilidad marcada a la insulina exógena ${ }^{25}$.

En relación a las características de los pacientes que presentaron hiperglicemia secundaria a L-asp, éstas fueron: edad $\geq 9,5$ años, IMC $\geq$ p 85 y antecedentes familiares de Diabetes Mellitus. La glicemia promedio observada fue de $160 \pm 225 \mathrm{mg} / \mathrm{dl}$. Se observó aparición de la RAM durante la fase de Inducción.

El promedio de edad fue $>9,5$ años en coincidencia con lo descrito en estudios previos ${ }^{15,19}$, en los cuales la mayoría de los episodios de hiperglicemia se presentaron en pacientes cercanos a la pubertad, debido a un incremento de la secreción de estrógenos y testosterona, ambas hormonas relacionadas con disminución de la tolerancia a la glucosa ${ }^{15,19,20,26}$. 
Otro factor conocido en la presentación de hiperglicemia asociada a L-asp, es el sobrepeso, descrito previamente como un factor de riesgo importante ${ }^{19,20}$. En esta serie, 4 de 6 pacientes con episodios categorizados como probables se encontraron en riesgo de obesidad (IMC $>$ p 85). Se ha comprobado que el tejido adiposo almacena lípidos y secreta numerosas hormonas, siendo el órgano endocrino de mayor tamaño del organismo, equivalente a 10$60 \%$ del peso total de un individuo según su composisción corporal e IMC ${ }^{27}$

En cuanto a los antecedentes familiares de Diabetes Mellitus, las investigaciones han contribuido a apoyar la teoría genética en la patogenia de ésta; algunas están enfocadas en el consejo genético y diseño de ensayos clínicos dirigidos a una futura prevención de la Diabetes Mellitus ${ }^{40,41}$. En este estudio al menos 3 de 6 pacientes tenían historia familiar de ella. Pui y cols. ${ }^{20}$ reportó una alta incidencia de hiperglicemia entre los pacientes con historia familiar de Diabetes Mellitus, similar a lo descrito años después por Roberson y cols. ${ }^{15}$, en un estudio en niños con LLA tratados con L-asp y corticoides, en el que el 50\% de los pacientes con hiperglicemia tuvieron antecedentes familiares de Diabetes Mellitus, apoyando la existencia de un factor genético asociado al desarrollo de hiperglicemia. Se debe tener en cuenta que al ser éste un estudio retrospectivo, basado en la recolección de datos de las historias clínicas, es posible que en algunos casos haya faltado información en la ficha con respecto a los antecedentes familiares, lo cual representa un sesgo que debe ser considerado.

En relación a otros fármacos que se administraron de forma concomitante al uso de Lasp, los resultados del presente estudio mostraron que todos los pacientes fueron tratados con corticoides, prednisona o dexametasona. En relación a la administración simultánea de corticoides y L-asp, no es posible discriminar los casos de hiperglicemia causados por corticoides de aquellos causados por L-asp. Los estudios y reportes de casos hacen alusión a la hiperglicemia producida por la asociación L-asp/corticoides, ya que se administran en forma combinada ${ }^{14-17,19,28}$ por esta razón es necesario mantener un alto índice de sospecha de RAM, mediante la pesquisa de síntomas y signos de hiperglicemia, con el fin de evaluar las sospechas de RAM con Algoritmo de Causalidad del Sistema Español de Farmacovigilancia, para su categorización.

En relación a la etapa del tratamiento, la mayoría de los episodios de hiperglicemia en las RAM probables, ocurrieron durante el protocolo de Inducción, lo que coincide con publicaciones previas en las que se describió episodios de hiperglicemia durante esta fase, mientras que en las siguientes, hubo discreto aumento de la glicemia o ésta se mantuvo en rango normal $1^{16,20,29,30}$.

Durante el protocolo de Inducción, la administración de L-asp de acuerdo al protocolo PINDA (2006) se usó en dosis de $5.000 \mathrm{UI} /$ $\mathrm{m}^{2}$ de superficie corporal, por 8 veces en 3 semanas, que es más frecuente y por tiempo más prolongado que en otras fases en las que se administra L-asp.

En cuanto al tratamiento de la hiperglicemia, 2 de los 6 pacientes categorizados como Probables recibieron tratamiento que consistió en régimen diabético en todos los casos, administración de hipoglicemiantes orales (metformina o glibenclamida) en algunos casos $\mathrm{y} / \mathrm{o}$ uso de insulina regular y/o insulina $\mathrm{NPH}$, en aquellos episodios en los que la glicemia superaba los $200 \mathrm{mg} / \mathrm{dL}$. En la literatura internacional se describe como medida terapéutica para el tratamiento de pacientes con hiperglicemia asociada a L-asp, la modificación de la dieta a un régimen diabético hipocalórico y líquidos, además de tratamiento con insulina regular, en la mayoría de los casos ${ }^{15,16,28,31-35}$. La duración del tratamiento descrito en la bibliografía, va desde unos pocos días hasta un año, teniendo estricta relación con el logro de normoglice$\mathrm{mia}^{28}$. En general, la literatura señala que debe ofrecerse un rápido tratamiento buscando la normoglicemia, de manera de prevenir eventos más graves como cetoacidosis diabética y coma hiperosmolar no cetoacidótico ${ }^{16,20}$, eventos adversos que no se presentaron durante este estudio.

En conclusión, la incidencia de hiperglicemia asociada al uso de L-asp fue $6,74 \%$, similar a lo descrito en la literatura internacional, siendo: edad $\geq 9,5$ años, riesgo de obesidad, 
antecedentes familiares de Diabetes Mellitus, tratamiento de Inducción y administración concomitante de corticoides, los factores asociados a su presentación. La información recolectada fue insuficiente para atribuir los episodios de hiperglicemia al tratamiento con L-asp, quedando categorizada como relación causal Probable de acuerdo al Algoritmo de Causalidad. La administración simultánea de corticoides en todos los casos permite atribuir los episodios de hiperglicemia a la asociación corticoides - L-asp, más que a uno u otro medicamento. El conocimiento de la frecuencia de las RAM asociadas a L-asp, permite intervenir sobre algunos de los factores de riesgo, como el de obesidad y mantener un alto índice de sospecha para su detección y tratamiento oportunos.

\section{Referencias}

1.- Oettgen H, Stephenson P, Schwartz M, Leeper R, Tallal $L$, Tan C: Toxicity of E. coli L-Asparaginase in Man. Cancer 1970; 25: 253-78.

2.- Narta U, Kanwar S, Azmi W: Pharmacological and clinical evaluation of L-asparaginase in the treatment of leukemia. Clinical Reviews in Oncology/Hematology 2007; 61: 208-21.

3.- Molineux G: Pegylation: Engineering improved biopharmaceuticals for oncology. Pharmacotherapy 2003; 23: 3S-8S.

4.- Kyonen M, Folatre I, Zolezzii P, Badilla V, Marín F: Reacciones adversas a L-asparaginasa en pacientes con leucemia linfoblástica aguda. Rev Med Chile 2006; 134: 1530-4.

5.- Barron A, Luke K, Hsu E, Torsher L, Deveber L, Cairney $A$ : Toxicity Study of Erwinia Asparaginase as First Line Therapy for Chilhood Acute Lymphocytic Leukemia. Hannnover. Proceedings of the 5th Pharmacology Workshop Satellite to XXIV SIOP Meeting 1992; 1004.

6.- Caruso V, Iacoviello L, Di Castelnuovo A, et al: Thrombotic complications in childhood acute lymphoblastic leukemia: a meta-analysis of 17 prospective studies comprising 1752 pediatric patients. Blood 2006; 108: 2216-22.

7.- Andrew M, Brooker L, Mitchell L: Acquired Antitrombina III Deficiency Secondary to Asparaginase Therapy in Childhood Acute Lymphoblastic Leukemia. Blood Coagulation and Fibrinolysis 1994; 15: 24-36.
8.- Müller H, Boos J: Use of L-asparaginase in childhood ALL. Critical Reviews in Oncology/Hematology 1998; 28: 97-113.

9.- Devita VT, Hellman SY, Rosenberg SA: Cancer: Principles \& Practice of Oncology, 3rd. JB Lippincott Company, Philadelphia, PA. 1989.

10.- Halton J, Nazir D, Mcqueen M, Barr R: Blood Lipid Profiles in Children with Acute Lymphoblastic Leukemia. Cancer 1998; 83: 379-84.

11.- Parsons S, Skapek S, Neufeld E, Kuhlman C, Young M, Donelly M: Asparaginase-Associated Lipid Abnormalities in Children with Acute Lymphoblastic Leukemia. Blood 1997; 89: 1886-95.

12.- Karabulut R, Sönmez K, Afsarlar C, Türkylmaz Z, Can basaklar A, Kale N: Pancreas Pseudocyst Associated with L-asparaginase Treatment: a Case Report. Acta Chir Belg 2005; 105: 667-9.

13.- Hsu Y, Chen Y, Ho C, Kao W, Chao T: Diabetic ketoacidosis and persistent hyperglycemia as long-term complications of L-asparaginase-induced pancreatitis. Zhonghua Yi Xue Za Zhi (Taipei) 2002; 65: 441-5.

14.- Roberson J, Raju S, Shelso J, Pui C, Howard S: Diabetic Ketoacidosis During Therapy For Pediatric Acute Lymphoblastic Leukemia. Pediatr Blood Cancer 2008; 50: 1207-12.

15.- Baillargeon J, Langevin A, Mullins $J$, et al: Transient Hyperglycemia in Hispanic Children With Acute Lymphoblastic Leukemia. Pediatr Blood Cancer 2005; 45: 960-3.

16.- Spinola-Castro A, Siviero-Miachon A, Andreoni S, Tosta-Hernández P, Macedo C, Lee M: Transient Hyperglycemia During Childhood Acute Lymphocytic Leukemia Chemotherapy: An Old Event Revisited. Clinical Advances in Hematology and Oncology 2009; 7: 465-72.

17.- Craig M, Hattersley A, Donaghue K: Definition, epidemiology and classification of diabetes in children and adolescents. Pediatr Diabetes 2009; 10: 3-12.

18.- Karch F, Lasagna L: Toward the operational identification of adverse drug reactions. Clin Pharmacol Ther 1977; 21: 247-54.

19.- Lowas $S$, Marks D, Malempati S: Prevalence of Transient Hyperglycemia During Induction Chemotherapy for Pediatric Acute Lymphoblastic Leukemia. Pediatr Blood Cancer 2009; 52: 814-8.

20.- Pui C, Burghen G: Risk factors for hyperglycemia in children with leukemia receiving L-asparaginase and prednisone. J Pediatr 1981; 99: 46-50.

21.- Sonabend R, Mckay S, Okcu M, Yan J, Haymond M, Margolin J: Hyperglycemia During Induction Therapy 
Is Associated With Increased Infectious Complications in Childhood Acute Lymphocytic Leukemia. Pediatr Blood Cancer. 2008; 51: 387-92.

22.- Ministerio de Salud Chile: Protocolo Cáncer Infantil. 2006.

23.- Vásquez F: Manejo de la hiperglucemia secundaria al tratamiento con corticoides. Av Diabetol 2006; 22: 1949.

24.- Braithwaite S, Barr W, Rahman A, Quddsi S: Managing diabetes during glucocorticoid therapy. How to avoid metabolic emergencies. Postgrad Med 1998; 104: 163-6.

25.- Levetan C, Magee M: Hospital management of diabetes. Endocrinol Metab Clin North Am 2000; 29: 745-70.

26.- Ortega J, Nesbit M, Donaldson M: L-Asparaginase, vincristine, and prednisone for induction of first remission in acute lymphoblastic leukemia. Cancer Res 1977; 37 : 535.

27.- Rocha V, Libby P: The multiple facets of the fat tissue. Thyroid. 2008; 18: 175-83.

28.- Alves $C$, Chaves $C$, Souza M: Diabetes Melito Transitório Relacionado à Terapia Com L-Asparaginase. Arq Bras Endocrinol Metab 2007; 51: 635-8.

29.- Jaffe $N$ : Diabetes mellitus secondary to L-asparaginase therapy. J Paediatr 1972; 81: 1220-1.

30.- Howard S, Pui C: Endocrine complications in pediatric patients with acute lymphoblastic leukemia. Blood Reviews 2002; 16: 225-43.

31.- Charan V, Desai N, Singh A: Diabetes mellitus and pancreatitis as a complication of L-asparaginase therapy. Indian Pediatr 1993; 30: 809-10.

32.- Gillette P, Hill L, Starling K, Fernbach D: Transient diabetes mellitus secondary to L-asparaginase therapy in acute leukemia. The Journal of Pediatrics 1972; 81:
10-11.

33.- Shenoy A, Nithyananda M, Aravindakshan R: Transient Diabetes Mellitus following L-Asparaginase Therapy. Kuwait Medical Journal 2007; 39: 188-9.

34.- Arranz A, Cabezudo M, Andia V, López A, Bárez A: Hiperglucemia grave con coma hiperosmolar y acidosis láctica inducida por L-asparraginasa y prednisona. Endocrinol Nutr 2003, 50: 348-50.

35.- Dundar B, Eren E, Oktem F, Dundar N, Tunc B, Canatan $D$ : Hyperosmolar non-ketotic syndrome in a child associated with L-asparaginase and prednisolone. Pediatrics International 2007; 49: 256-7.

36.- Avramis $V$. and Tiwari P: Asparaginase (native ASNase or pegylated ASNase) in the treatment of acute lymphoblastic leukemia. International Journal of Nanomedicine 2006; 1: 241-54.

37.- Baudrand R, Arteaga E, Moreno M: El tejido graso como modulador endocrino: Cambios hormonales asociados a la obesidad. Rev Med Chile 2010; 138 : 1294-301.

38.- De Pablos P, Martínez F: Significado clínico de la obesidad abdominal. Endocrinol Nutr 2007; 54: 265-71.

39.- Asenjo S, Muzzo BS, Pérez MV, Ugarte PF, Willshaw $M E$ : Consenso en el diagnóstico y tratamiento de la diabetes tipo 1 del niño y del adolescente. Rev Chil Pediatr 2007; 78: 534-541.

40.- Gillespie KM, Gale EA, Bingley PJ: High familial risk and genetic susceptibility in early onset childhood diabetes. Diabetes 2002; 51: 210-4.

41.- Sipetic S, Vlajinac H, Kocev N, Marinkovic J: The Belgrade childhood diabetes study: a multivariate analysis of risk determinants for diabetes. European Journal of Public Health 2005; 15: 117-22. 